herangezogene Versagungsgrund die Ablehnung des Antrags nicht trägt und die Genehmigung nach dem bis zum Zeitpunkt der Entscheidung gewonnenen Erkenntnisstand nicht schon aus anderen Gründen offensichtlich $\mathrm{zu}$ versagen ist, vgl. OVG Münster, Urt. v. 19.6.2007 - 8 A 2677/06, NWVB1. 2008, 26, im Anschluss an BVerwG, Urt. v. 14.4.1989 - 4 C 52.87, NVwZ 1990, 257; OVG Koblenz, Urt. v. 11.5.2005 - 8 A 10281/05, BauR 2005, 1606.

Das ist hier der Fall. (wird ausgeführt) ...

DOI: 10.1007/s10357-012-2230-3

\section{Zugang zu Umweltinformationen der DB Netz AG} UIG §3

Bei der DB Netz AG handelt es sich um eine informationspflichtige Stelle i.S.d. $\$ 2$ Abs. 1 Nr. 2 UIG.

VG Frankfurt, Beschluss vom 7. 6.2011 - 7 K 634/10.F-

Nachdem die Beteiligten die Hauptsache in der mündlichen Verhandlung übereinstimmend für erledigt erklärt haben, ist gemäß \161 Abs. 2 VwGO nach billigem Ermessen und unter Berücksichtigung des bisherigen Sach- und Streitstandes über die Kosten des Verfahrens zu entscheiden.

Nach dem gegenwärtigen Sach- und Streitstand des erledigten Verfahrens, der der Kostenentscheidung zugrunde zu legen ist, hat die Beklagte die Kosten des Verfahrens zu tragen.

Zunächst steht dem Kläger gemäß $₫ 3$ Abs. 1 UIG i.V.m. $\int 4$ Abs. 1, Abs. 2 Satz 4 UIG ein freier Zugang zu Umweltinformationen ohne Darlegung eines rechtlichen Interesses auf Antrag zu. Da $\$ 6$ Abs. 2 Satz 1 UIG ein Widerspruchsverfahren nur dann vorsieht, wenn gegen die Entscheidung durch eine Stelle der öffentlichen Verwaltung i.S.d. $\$ 2$ Abs. 1 Nr. 1 UIG vorgegangen wird, ist der gegen eine juristische Person des Privatrechts wie der Beklagten gemäß $₫ 2$ Abs. 1 Nr. 2 UIG begehrte Informationszugangsanspruch im Wege der Leistungsklage zu verfolgen (Landmann/Rohmer, Umweltrecht, 56. Aufl. 2009, 96 Rdnr. 15).

Die vom Kläger begehrten Daten dürften auch Umweltinformationen gemäß $\$ 2$ Abs. 3 UIG darstellen. So begehrt der Kläger zunächst Auskunft über den Bestand und sodann Akteneinsicht in Unterlagen, die geeignet sind, Umweltinformationen $\mathrm{zu}$ enthalten wie z.B. Bau- und Betriebsunterlagen, Planfeststellungsunterlagen und Kosten-NutzenAnalysen. Weiter begehrt er Zugang zu Daten, die selbst Umweltinformationen darstellen, wie emissions- und immissionsrelevante Gutachten, Dokumentationen, Berichte und Messwerte über Emissionen und Immissionen.

Bei der Beklagten ... AG handelt es sich nach Auffassung der Kammer auch um eine informationspflichtige Stelle i. S.d. $\$ 2$ Abs. 1 Nr. 2 UIG. Danach sind informationspflichtige Stellen auch juristische Personen des Privatrechts, soweit sie öffentliche Aufgaben wahrnehmen oder öffentliche Dienstleistungen erbringen, die im Zusammenhang mit der Umwelt stehen, insbesondere solche der umweltbezogenen Daseinsvorsorge und dabei der Kontrolle des Bundes oder einer unter der Aufsicht des Bundes stehenden juristischen Person des öffentlichen Rechts unterliegen.

Die Beklagte erbringt eine öffentliche Dienstleistung, nämlich Verkehrsdienstleistungen, in dem sie das Schienennetz bereit stellt und betreibt. Sie versorgt die Bevölkerung mit Verkehrsinfrastruktur und Verkehrsangeboten und betreibt damit eine gemeinwohlerhebliche Daseinsvorsorge. Diese ist nach Auffassung der Kammer auch umweltbezogen bzw. steht im Zusammenhang mit der Um- welt. Eine weitere Auslegung lässt dabei ausreichen, dass die Tätigkeit Auswirkungen auf die Umwelt hat, was zur Bejahung der umweltbezogenen Daseinsvorsorge führen würde, da die Existenz und der Betrieb des Schienenwegenetzes zu Veränderungen des Bodens- und Landschaftsbildes führt, Auswirkungen auf Flora und Fauna hat und auch eine Lärmquelle für Menschen darstellt. Diese Auslegung hat den Wortlaut des $\$ 2$ Abs. 1 Nr. 2 UIG für sich. Die Beklagte bezieht sich dementgegen auf den Wortlaut von Art. 2 Nr. 2 lit. g der Richtlinie 2003/4/EG des Europäischen Parlaments und des Rates vom 28. Januar 2003 über den Zugang der Öffentlichkeit zu Umweltinformationen und zur Aufhebung der Richtlinie 90/313/EWG des Rates (UIRL 2003/4), wonach juristische Personen, die im Zusammenhang mit der Umwelt öffentliche Zuständigkeiten haben, öffentliche Aufgaben wahrnehmen oder öffentliche Dienstleistungen erbringen, im Sinne dieser Richtlinie als „Behörde“ gelten. Danach käme es maßgeblich darauf an, ob die juristische Person des Privatrechts Aufgaben des Umweltschutzes wahrnimmt. Nach Auffassung der Kammer ist in Anlehnung an $\$ 2$ Abs. 3 UIG, der einen weiteren Begriff der Umweltinformationen zugrundelegt, darauf abzustellen, ob die von der Beklagten erbrachte Dienstleistung im funktionellen und historischen Zusammenhang mit dem Umweltschutz gesehen wird, wobei es nicht auf die Erheblichkeit der mit der Aufgabe oder Dienstleistung verbundenen Umweltauswirkung ankommt. Für bestimmte Bereiche der Daseinsvorsorge ist ein Zusammenhang zwischen der öffentlichen Dienstleistung und dem Umweltschutz normativ geregelt. So bezweckt z.B. das Energiewirtschaftsgesetz in seinem $\$ 1$ Abs. 1 u.a. eine ,umweltverträgliche" Versorgung mit Elektrizität und Gas. Für Bahndienstleistungen enthält das Allgemeine Eisenbahngesetz keine solche Zweckbestimmung, während z. B. das Niedersächsische Nahverkehrsgesetz nach seinem $\$ 2$ Abs. 1 auch ,,im Interesse verträglicher Lebens- und Umweltbedingungen“" erging. Dies zeigt, dass der Begriff des Zusammenhangs mit der Umwelt nicht konstitutiv vom Gesetzgeber festgelegt wird. Er ist vielmehr nach allgemeinen Auslegungskriterien zu bestimmen, wonach dem Wortlaut des $\$ 2$ Abs. 3 UIG nach der Begriff, „Umwelt" weit zu verstehen ist. Der historische und funktionelle Zusammenhang der erbrachten Daseinsvorsorge mit dem Umweltschutz ist $\mathrm{zu}$ bejahen für private Rücknahmesysteme wie ... AG (...), Flughafenbetreiber und die ... AG sowie ggf. auch Tochterunternehmen der ... AG im Bereich der öffentlichen Daseinsvorsorge (vgl. Informationsrechte, Öffentlichkeitsbeteiligung und Rechtsschutz im Umweltrecht; Aarbus-Handbuch, Schlacke, Schrader, Bunge, 1. Auflage 2009; Reidt/Schiller in Landmann/Rohmer, Umweltrecht III, $\$ 2$ UIG Rdnr. 29 f.).

Die Beklagte unterliegt auch der Kontrolle des Bundes bzw. einer unter Aufsicht des Bundes stehenden juristischen Person des öffentlichen Rechts. Gemäß Art. 87e Abs. 3 Grundgesetz - GG - sind Eisenbahnen vom Bund als Wirtschaftsunternehmen zu führen. Dies ist durch eine Organisations- und nicht eine Aufgaben- und Kapital-Privatisierung erfolgt. $₫ 8$ des Allgemeinen Eisenbahngesetzes - AEG - regelt die rechtliche Verselbständigung der Eisenbahnen und macht inhaltliche Vorgaben zur Geschäftsführung. Die Beklagte unterliegt dabei einer umfassenden staatlichen Kontrolle durch das Eisenbahnbundesamt, welches die Eisenbahnaufsicht ausübt (vgl. hierzu auch BGH, Beschl. v. 9.12.2010 - 3 StR 312/10).

Darüberhinaus hat die Beklagte den Kläger klaglos gestellt, weshalb auch unter diesem Gesichtspunkt die Kostentragung durch die Beklagte sachgerecht ist. 